\title{
Metaheuristic Search Procedure based on Fuzzy Graph for $p$-Facility Problems
}

\author{
Wesley G. Almeida, Ernesto Araujo, Member, IEEE
}

\begin{abstract}
A metaheuristic search procedure modified by fuzzy logic and fuzzy set theory for solving $p$-facility location problems is proposed in this paper. Known as $p$-median problem, as well, the objective is to find $p$ centers (locations) in a network in order to minimize (maximize) the average distance between demand nodes and their medians. The proposed approach employs Greedy Randomized Adaptive Search Procedure (GRASP) modified by grading the distances from locations weighted by membership functions or fuzzy rules. In so doing, a novel approach named Fuzzy Greedy Randomized Adaptive Search Procedure (FUGRASP) is presented. In order to verify the feasibility of the proposed method, $p$ centers are computed to minimize the average distances among cities according to their respective air-traffic importance.
\end{abstract}

\section{INTRODUCTION}

The $p$-facility location problem is related to the selection of locations where any sort of facility, such as schools, health centers, hospitals, storages, factories, and so forth, has the average distance for a set of distributed demand nodes minimized (maximized). The $p$-facility location problem belong to the NP-hard problem class [1] mostly demanding huge effort and being time consuming when determine the location of the nodes by try-and-error approach, or even when expertise of specialists is employed. Finding out the best solution may require a large computational time, as well.

The complexity and the number of nodes as well as the demand of clients are factors that greatly affect the location of the $p$ centers. In order to attenuate the efforts to minimize (maximize) the average distance, the use of graphs is an alternative in the search of a solution of $p$-facility locations. This paper aims at suggesting a metaheuristic solution based on fuzzy graph in helping to determine the best solution, i.e., the best $p$-facility location.

The use of fuzzy graph applied to $p$-facility location problem, however, is an incipient approach that has just blossom up in the scientific research. This paper contributes in pushing forwards the boundaries of operational research by using fuzzy set theory, fuzzy logic, and approximate

Wesley G. Almeida is with Computer Sci. and Applied Math. Associated Lab. - LAC, at Instituto Nacional de Pesquisas Espaciais (INPE), Av. Astronautas, 1758, 12.227-010, São José dos Campos, SP, Brazil. (email: wesgomes2005@gmail.com)

Ernesto Araujo is with Integration and Testing Laboratory (LIT) and Space Engineering and Technology (ETE), at Instituto Nacional de Pesquisas Espaciais (INPE), Av. Astronautas, 1758, 12.227-010, São José dos Campos, SP, Brazil; with Health Informatics Department (DIS), at Universidade Federal de São Paulo (UNIFESP), R. Botucatu, 862, 04023-062, São Paulo, SP, Brazil; with the Hospital Municipal Dr. Jose de Carvalho Florence (HMJCF), Av. Saigiro Nakamura, 800, 04023-062, São José dos Campos, SP, Brazil; and Assoc. Paulista para o Desenvolvimento da Medicina (SPDM), R. Napoleão de Barros, 715, 04024-002, São Paulo, SP, Brazil. (email: ernesto.araujo@\{unifesp.br,lit.inpe.br $\}$ ) reasoning in order to improve search mechanisms for finding out better (optimal) solutions. The $p$-facility location problem in fuzzy graph consists in the problem with graphs that contain degrees of certainty (membership functions) to represent the imperfect knowledge concerning inherent uncertainties, imprecision, vagueness, and partial truth related to its edges, nodes, or parameters such as cost, capacity, and demand.

This paper aims at using fuzzy logic and fuzzy set theory intertwined with the metaheuristic Greedy Randomized Adaptive Search Procedure (GRASP) for the $p$-facility location problem. When employing these techniques in synergy, a Fuzzy Greedy Randomized Adaptive Search Procedure emerges. This new approach is employed here to compute $p$ centers in order to minimize the average distances among cities selected according to their respective air-traffic importance to show the feasibility of the proposed approach.

\section{Greedy Randomized Adaptive SeArch PROCEDURE MODIFIED BY FUZZY GRAPH}

\section{A. Greedy Randomized Adaptive Search Procedure (GRASP)}

The Greedy Randomized Adaptive Search Procedure (GRASP) can be defined as the iterative procedure composed of two phases. The first phase is denominated construction while the second is local search [2], [3], [4]. The pseudo code for GRASP algorithm is presented in Fig. 1.

In the construction phase, a feasible solution is build iteratively, element by element, until a solution is complete. The candidate elements, $d_{i}$, that compose the solution are ranked in a list, assigned candidate list. This list is ranked by using a greedy procedure named in the pseudo-code, ConstructGreedRandomizedSoln that measures the benefits that each element contributes to the whole solution. For greedy is understood the search for the best candidates in the list, i.e., at the top of the list.

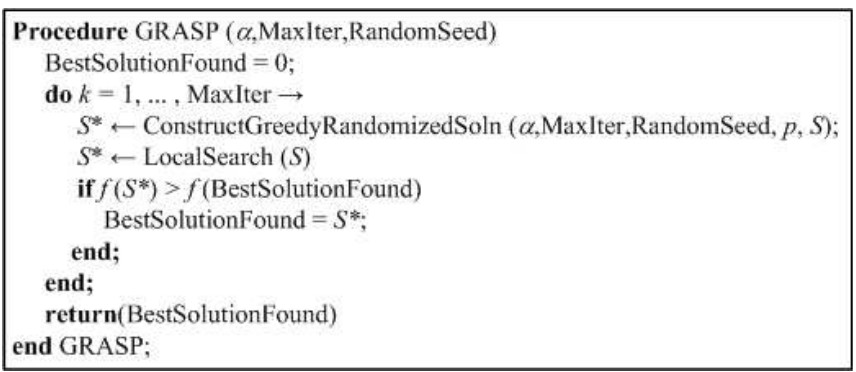

Fig. 1. Pseudo code for Greedy Randomized Adaptive Search Procedure. 
Each iteration is composed of three subclasses [13], [14]:

i) Construction of the Restrict Candidate List ( $R C L)$, that contain a reduced set of candidate elements that belong to the solution;

ii) Random Selection of elements within $R C L$ and their inclusion in the solution;

iii) Adaptation or a new computing of the greedy function for those elements that do not belong to the list yet.

The best solution determined for all performed GRASP iterations is the final solution result. The metaheuristic is adaptive due to the benefits associate to each element are upto-date in each iteration. This adaptive behavior is employed to incorporate the changes concerned to the selection of previous elements.

The subset $R C L$ is composed of the best elements within the candidate list. The control parameters, $|R C L|$, related to the size of the list define the search mechanism. When $|R C L|=1$, the solution mechanism refer to a purely greedy behavior of the algorithm. In this case, the selected element is always the best candidate meaning the one in the top of candidate list (greedy selection). In the other hand, when $|R C L|=n, \forall 0<n<1$, there is a random behavior for the solution mechanism. When the random selection prevail for an element within the $R C L$, it is obtained by using a probabilistic approach allowing that different, good solutions be generate but that are not optimal. Although this solution is not the best (optimal) - i.e., the greedy solution - for each iteration, this behavior presents the advantage of avoiding local minima in the search mechanism. The mean value and the variance of the fitness function for the solutions are directly influenced by $|R C L|$. Low values for $|R C L|$ presents a smaller variance since there is a reduced search space for the solution to be determined and so there is a higher chance of the search be trapped in a local minima (poor optimal local). In contrary, the higher is the $|R C L|$, the greater is the variance, thus, allowing a reduced chance of being trapped in a minimal local but with a increased number of iterations with sub-optimal solutions and greater are the neighborhood explored. Notice, then, that the main parameter to be setup when employing GRASP is the cardinality of $R C L$.

The effective application of this method requires, thus, the determination of an adequate size for $|R C L|$ in order to equilibrate the relation among $(i)$ quality of the solutions, (ii) number of iterations, and (iii) explored neighborhood [3], [4]. This trade-off aims at finding the optimal or suboptimal solution in a reduced time and computational cost. In turn, the local search may be carried out by a family of techniques that search the solution space changing from a solution to another in the neighborhood in order to select the best solution in the neighborhood.

In the local search the GRASP uses the initial solution obtained in the construction phase and explore the neighborhood close to this solution. If there is an improvement, the current solution is up-to-date and a new investigation in the neighborhood is carried out. The process repeat until no improvement is achieved. In the local search is necessary (a) determine an adequate neighborhood; (b) employ efficient data structures in order to increase the speed of convergence; (c) start with a good initial condition (close to a local optimal), that drive to an efficient local search [5].

\section{B. Fuzzy Greedy Randomized Adaptive Search Procedure}

In the Fuzzy Greedy Randomized Adaptive Search Procedure (FUGRASP) proposed in this paper, the construction phase is equivalent to the same phase in the classical greedy algorithm. The proposed approach differentiate from the latter, however, for each candidate solution, $d_{i}$, be assigned a degree of certainty according to the fuzzy set theory or IF-THEN fuzzy rules.

Fuzzy sets may be understood as the generalization of classical sets. A classical set, $A$, in a universe of discourse, $X=\left\{x_{1}, x_{2}, \ldots\right\}$, is defined by a characteristic function (mapping), $\mu_{A}(x)$, that assumes a null value for all elements in $X$ that does not belong to the set $A, \mu_{A}(x)=0 \forall x \notin A$, and an unitary value for those elements that belong to it, $\mu_{A}(x)=1$ if $x \in A$, that is, $\mu_{A}(x): X \rightarrow\{0,1\}$. If the classical set theory establishes that an element may belong, or not, to a set; a fuzzy set, $A$, in a universe of discourse, $X=\left\{x_{1}, x_{2}, \ldots\right\}$, is defined by a membership function, $\mu_{A}(x): X \rightarrow[0,1]$, mapping each element, value or point, $x$, in the domain, $X$, to a number (degree) in the interval between the real numbers $[0,1]$. It means that elements may belong partially to a set. The set of ordered pairs of an element, $x$, such that $A=\left\{\left(x, \mu_{A}(x)\right) \forall x \in X\right\}$, where $\mu_{A}(x)$ is known as membership degree of $x$ in $A$ [6]. The membership function, $\mu_{A}(x)$, may also be understood as the compatibility degree between an element, $x$, and the concept expressed by $A$.

Thus, the proposed FUGRASP advocates that instead of selecting the best candidate, $d_{i}$, by using the classical set theory that assume dichotomic values, $\mu\left(d_{i}\right)=\{0,1\}$, the candidate is weighted by gradual values, $\mu\left(d_{i}\right)=[0,1]$. The advantage of employing fuzzy sets is the use of categories with flexible edges, of progressive values from one property to another, or the use of approximate values. Thus, when a fuzzy set is employed it is allowed an element within the candidate list to assume gradual membership values. In so doing, the candidate list becomes a fuzzy candidate list encompassing a list of candidate solutions with degrees of certainty determined by the FUGRASP.

The pseudo-code for the novel FUGRASP algorithm is shown in Fig. 2. Notice that the distances among nodes received gradual values obtained from membership functions after the construction phase is performed. There are distinct manners to set the architecture of FUGRASP. One alternative to be employed in this paper is shown in Fig. 3. These membership functions are distributed upon exogenous parameters, $\theta$, that may be related, for example, to confidence, safety, and so forth representing uncertainty, imprecision, vagueness of the information. The distance among the nodes is now on weighted by a degree of certainty driven by an exogenous 


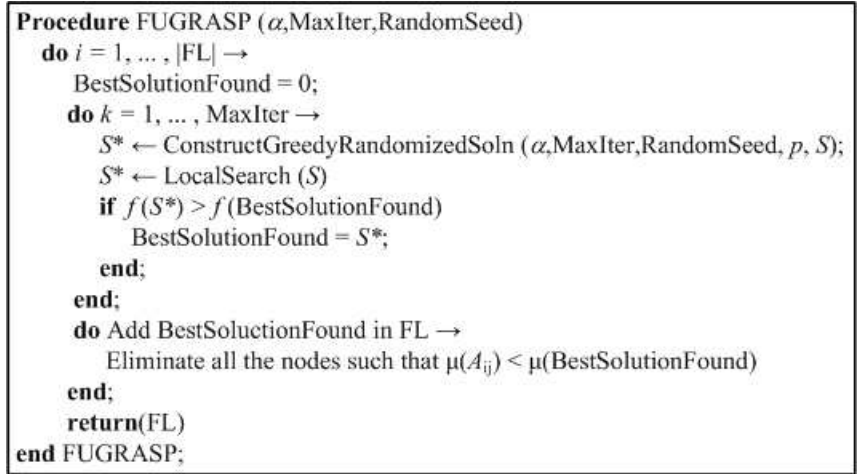

Fig. 2. Pseudo code for Fuzzy Greedy Randomized Adaptive Search Procedure - FUGRASP.

factor (control parameter), $\theta$, and represented as follows:

$$
\tilde{d}=d(\theta), \forall i=1, \ldots, n \text {. }
$$

According to the proposed approach, the universe of discourse may be parted by a single membership function, or a set of membership functions (in this case, representing classes), as depicted in Fig. 3. In the presence of diverse membership functions, IF-THEN fuzzy rules may be employed allowing another degree of decision in the metaheuristic search procedure.

The degree of activation of the membership function from the feasible solution candidate in the construction phase is determined by using the conjunction operation when an operator from the family of Triangular norm (T-norm) is selected. Although any T-norm may be employed the most usual found in literature are the minimal operator:

$$
\mu\left(\tilde{d}_{i j}\right)=\min \left[d_{i j}^{1}(\theta), d_{i j}^{2}(\theta), \ldots, d_{i j}^{m}(\theta)\right] .
$$

where $m$ corresponds to distinct membership functions, or the algebraic product operator:

$$
\mu\left(\tilde{d}_{i j}\right)=\prod_{m=1}^{n} d_{i j}^{m}(\theta)
$$

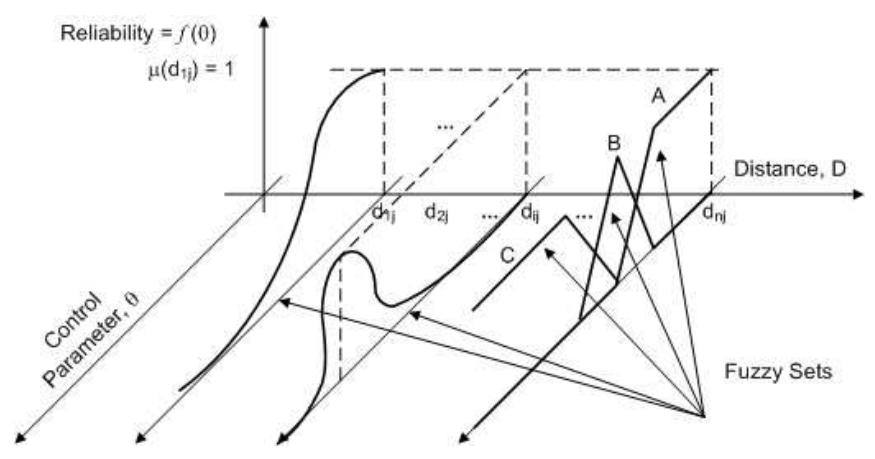

Fig. 3. Membership functions for grading the reliability degree (certainty) for the candidate elements related to the distances between two extremes of nodes.

\section{FUZZY GRAPH APPLIED TO THE P-FACILITy LOCATION PROBLEM}

In this paper, the Fuzzy Greedy Randomized Adaptive Search Procedure (FUGRASP) is employed for determining the best feasible solution for $p$-facility location problems. The example hereafter has the objective of demonstration the feasibility of the proposed FUGRASP when facing the minimization of the average distances among cities taking into account the air-traffic importance.

\section{A. General Description of P-Facility Location Problems}

The problem of $p$-facility location problem is described according to the binary integer programming model, given as:

$$
f(x)=\min \sum_{i} \sum_{j} C_{i j} x_{i j}
$$

subject to:

$$
\begin{gathered}
\sum_{k} x_{k k}=p, \\
\sum_{k} x_{i k}=1, \forall i \in V, \\
x_{k k}-x_{i k} \geq 0, \forall i, k \in V, \\
x_{i k} \in\{0,1\} \geq 0, \forall i, k \in V,
\end{gathered}
$$

where $C_{i j}$ is the cost of displacement in the edge, from the $i$-th to the $j$-th node, and $x_{i k}$ is a decision variable, such that $x_{i k}=1$ whether the $i$-th node is allocate at the median value located in the $k$-th node, or $x_{i k}=0$, in contrary. When $x_{k k}=1$ it means that the $k$-th node is the median. It is worth mentioning that the cost is not necessarily related to distance, being related to innumerous other parameters. When dealing with distance, the cost and the distance are equivalent, $C_{i j}=$ $d_{i j}$. In so doing, expression in (4) becomes:

$$
f(x)=\min \sum_{i} \sum_{j} d_{i j} x_{i j}
$$

\section{B. P-Facility Location Problems adapted to FUGRASP}

According to the novel FUGRASP approach, each cost (distance), $d_{i j} \in D$, is weighted by the activation degree of a membership function, $\mu_{A_{i}}$, distributed in a new universe of discourse that corresponds to the control parameter, $\theta$, as presented in Fig. 3. Hence, the expression in (4) or (9) is modified to receive this adaptive, weighted value obtained from the membership function, that is, respectively:

$$
f(x)=\min \sum_{i} \sum_{j} C_{i j}(\theta) x_{i j}
$$

or

$$
\begin{array}{r}
f(x)=\min \sum_{i} \sum_{j} d_{i j}(\theta) x_{i j} \\
=\min \sum_{i} \sum_{j} \tilde{d}_{i} x_{i j}
\end{array}
$$

subject to the same constraints in (5), (6), (7), and (8). 
In this formulation, the fitness function (4) establish the displacement cost that must be the lower possible, the constraints given by (5) fix the number $p$ of facilities to be located, the constraints given by (6) guarantee that each demand node is allocate a single center, the constraints given by (7) assure that the locations are performed only for the nodes that are medians, and finally the constraints in (8) correspond to the conditions of integrality of the decision variables. The variable $\theta$ corresponds to the firing degree (the degree of activation) of the membership functions referents to an exogenous parameter that weights the costs.

\section{Airport Location Problem and the Air-traffic Flow of Passengers}

The efficiency of the proposed FUGRASP metaheuristic search method is carried out upon an air-traffic database from Civil Aeronautics Board (CAB). The CAB database is based on the air-traffic flow of passengers taken into account 25 cities selected according to their air-traffic importance in 70's [7]. This database is chosen for evaluating mechanism due to it has became a standard when interested in verifying the effectiveness of algorithms for $p$-facility problems by employing classical graphs, in general, or when dealing with fuzzy graphs, in particular [8], [9], [10], [11], [12]. Due to that, the objective is to find out airport locations in order to minimize the cost of the air-traffic network.

For the p-median problem adopted here, distances from one city to another, $d_{i j}$, refer to the edges. The air-traffic flow of passengers, $W_{i j}$, is associated to the exogenous factor (control parameter), $\theta=W_{i j}$, for achieving the activation degree of the membership functions, $\mu\left(W_{i j}\right)=P_{i j}$. In this example, the highest flow among all the edges is given by $W_{\max }$ that corresponds $P_{i j}=0$ since it is assumed that a huge traffic of passengers increases the possibility of problems. It is worth mentioning that, other classes of problem could take into account the contrary representation

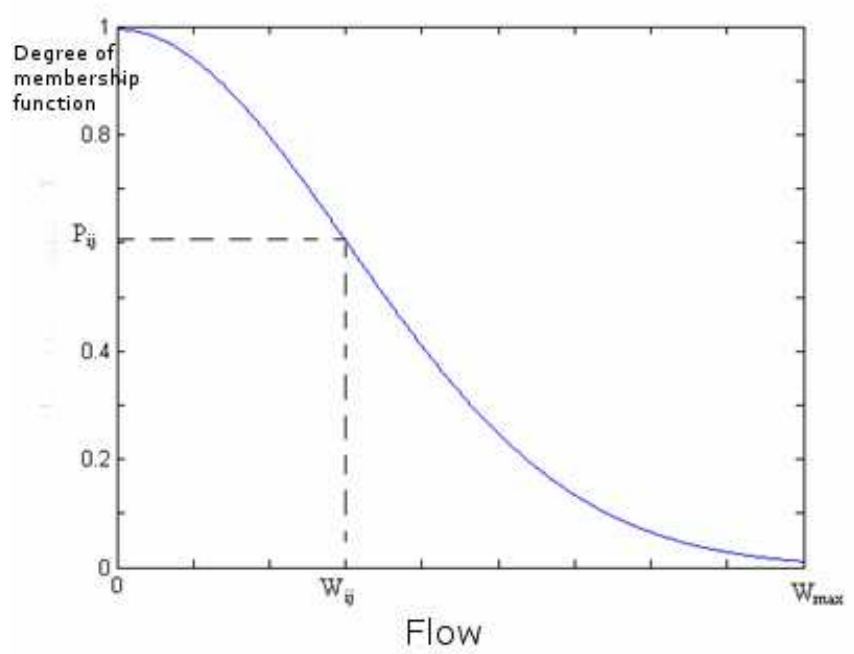

Fig. 4. Membership degree of edges, $M_{i j}$.
TABLE I

RESUlts OBTAINED WITH FUGRASP.

\begin{tabular}{c|c|c|c|c}
\hline \hline $\mathbf{N}$ & $\mathbf{P}$ & Cost, $f\left(x_{i j}\right)$ & Firing Degree, $\mu_{A(\theta)}$ & Time \\
\hline \hline \multirow{3}{*}{5} & \multirow{3}{*}{3} & 624,56 & 0,26 & 0,35 \\
& & 743,34 & 0.54 & 0,53 \\
& & 802,92 & 0,92 & 0,91 \\
\hline \multirow{3}{*}{10} & \multirow{3}{*}{3} & 2281,60 & 0,12 & 0,50 \\
& & 2348,51 & 0,13 & 0,50 \\
& & 2936,27 & 0,53 & 0,49 \\
\hline \multirow{2}{*}{15} & \multirow{3}{*}{3} & 5426,07 & 0,27 & 0,65 \\
& & 5553,68 & 0,29 & 0,65 \\
& & 5600,32 & 0,38 & 0,63 \\
\hline \multirow{3}{*}{20} & \multirow{3}{*}{3} & 6728,99 & 0,65 & 0,77 \\
& & 6764,21 & 0,66 & 0,76 \\
& & 6893,93 & 0,85 & 0,77 \\
\hline \multirow{2}{*}{25} & \multirow{3}{*}{3} & 9435,66 & 0,40 & 0,91 \\
& & 9630,08 & 0,65 & 0,91 \\
& & 9664,24 & 0,89 & 0,89 \\
\hline \hline
\end{tabular}

as well as other membership functions as depicted in Fig.3.

In order to illustrate the FUGRASP, all the membership functions assume the Gaussian shape (Fig. 4) computed as:

$$
\mu_{d}(x, \sigma(\theta), c)=\exp \left\{\frac{-(x-c)^{2}}{2 \sigma(\theta)^{2}}\right\},
$$

where the position given by the center (core) of the function, $c$, and the shape given by the spread (support), $\sigma$. In this case, the support is modified according to an exogenous parameter, $\theta$, related to the air-traffic flow, as previously mentioned. The center of the membership function occurs when $W_{i j}=0$, i.e., there is the maximum value of the activation degree (Fig. 4). In this example, without any lack of generality, the parameter $m=1$ in (2) or (3) since it is chosen one membership function for illustrating the use of FUGRASP.

As the distance increases the reliability of the distance decreases, for instance influenced by the cost, roads/street conditions, safety, risks of any sort etc. In this paper, the reliability decreases according to the air-traffic flow of passengers. Hence, an edge with low flow is considered more reliable for the solution. The lower is the flow that exists in this edge, the higher is the membership degree attributed to this information.

Results obtained with such an example are shown in Table I. Distinct number of cities, $N$, are employed to validate the proposed method. The number of airports for satisfying the air-traffic requirements for the set of $N$ cities is given by $P$. This table presents the air-traffic network cost and the firing degree (activation degree of membership function) representing the reliability associated to the airtraffic flow of passengers. Additionally, it is presented the running time for achieving such results. Different from the GRASP solution that supplies only one result (cost), while employing the FUGRASP there is the advantage of finding out distinct results (costs). Each of these costs is associated to a degree of reliability derived from an exogenous factor (control parameter) that would not be taking into account with the classical approach. There are as many costs as the number of air-traffic flow of passengers. Nevertheless, in 
Table I, for each set of cities, there is just three results for illustrative example. In a practical situation, hence, the decision maker (manager, administrative staff etc.) may count on three options for selecting an appropriate choice (decision) for each set of cities. For instance, one of the criteria is based on the solution that presents the greater (lower) degree of reliability (risk) according to the problem one is facing off.

\section{CONCLUSION}

Problems of combinatory optimization employing parameters related to fuzzy set theory and fuzzy logic may achieve a great relevance when dealing with applications in the real world. Costs may generate uncertainties, imprecision, vagueness and partial truth due to the great variation in prices, in such a way that these imperfect values for parameters in the combinatory optimization may be characterized as fuzzy sets. The better are the solutions for the problem higher are the economical profits for the sectors in which the problem is inserted in.

The $p$-facility location problem comprise the combinatory optimization and become an interesting approach to be characterized by fuzzy set theory and fuzzy logic. This paper combines fuzzy approach to the Greedy Randomized Adaptive Search Procedure (GRASP) yielding a novel search mechanism denominated Fuzzy Greedy Randomized Adaptive Search Procedure (FUGRASP). The advantage of such a method is related to the possibility of including those imperfect knowledge concerning the edges, nodes, and demands as membership functions. Taking into account the distribution and the amount of membership functions, IFTHEN fuzzy rules may also be employed to adding up levels of decision to the $p$-facility location problem.

In order to validate the proposed novel algorithm, membership functions related to fuzzy parameters assume distinct values as function of an exogenous parameter, in this case, the air-traffic flow. In this example, the objective is to minimize the sum of distances from a demand node and its mean among cities chosen according to their respective air-traffic importance. Results obtained show the feasibility of the proposed search mechanism. It is worth mentioning that as occur with any metaheuristic solution the proposed approach does not guarantee the solution be optimal for the problem. Nevertheless, according to the prompted results for the experiments carried out in this paper the fuzzy greedy randomized adaptive search procedure is able to generate adequate solutions. Further, in a reduced computational time with the advantage of introducing the inherent uncertainties, imprecision, vagueness, and partial truth of the information used in the combinatory optimization procedure.

\section{REFERENCES}

[1] M. R. Garey and D. S. Johnson, "Computers and Intractability. A Guide to the Theory of NP-Completeness, W. H. Freeman and Company, 2007.

[2] M. G. C. Resende, Greedy Randomized Adaptive Search Procedures (GRASP), AT\&T Labs Research Technical, 1998, Report: 98.41.1..

[3] M. G. C. Resende and C. C. Ribeiro, Greedy Randomized Adaptive Search Procedures (GRASP), AT\&T Labs Research Technical, 2001.

[4] M. G. C. Resende and C. C. Ribeiro, Handbook of Metaheuristics, "Greedy randomized adaptive search procedures", London: Kluwer Academic Publishers, F. Glover and G. Kochenberger, eds. pp.219-249, 2003.

[5] M. L. Rocha and C. L. Junior and C. M. P. Barros, "Aplicao de uma Heurstica GRASP Paralela ao Problema da P-Mediana", Proc. X Encontro de Iniciao Cientfica do Instituto Tecnolgico de Aeronutica, São José dos Campos, ITA, 2004.

[6] Bellman, R. E. and Zadeh, L. A., "Decision-Making in a Fuzzy Environment", Management Science, vol.17, n.4, supp.B, pp.141-164, 1970.

[7] M. O'Kelly, "A quadratic integer program for the location of interacting hub facilities", European Journal of Operational Research, vol.32, pp.393-404, 1987.

[8] J. F. Chen, "A hybrid heuristic for the uncapacited hub location problem", The International Journal of Management Science, vol.35, pp.211-220, 2007.

[9] C. B. Cunha and M. R. Silva, "A genetic algorithm for the problem of configuring a hub-and-spoke network for a LTL trucking company in Brazil", European Journal of Operational Research, vol.179, pp.747758, 2007.

[10] M. Sasaki and M. Fukishima, "On the hub-and-spoke model with arc capacity constraints", Journal of the Operations Research Society of Japan, vol.46, n.4, pp.409-428, 2003.

[11] M. R. Silva, "Uma contribuio ao problema de localizao de terminais de consolidao no transporte de carga parcelada", Escola Politcnica da Universidade de So Paulo, São Paulo, MSc Dissertation, 2004.

[12] H. Topcuogly and F. Corut and M. Ermis and G. Yilmaz, "Solving the uncapacitated hub location problem using genetic algorithms", Computers and Operations Reseach, vol.32, pp.967-984, 2005.

[13] C. D. S. Morelli and L. T. Vieira, "Análise de Métodos Heurísticos de Característica Gulosa", IV Semana Acadêmica do Programa de PósGraduação em Computação, São José dos Campos, 1999.

[14] M. J. F. Souza, "Programao de horrios em escolas: uma aproximao por metaheursticas", Universidade Federal do Rio de Janeiro (UFRJ), Rio de Janeiro, PhD Thesis, 2000. 